\section{Cuantificación económica de los beneficios en salud asociados a la reducción de la contaminación por $\mathrm{MP}_{10}$ en Concepción Metropolitano, Chile}

\author{
CRISTIAN MARDONES ${ }^{1, \mathrm{a}}$, ANDRÉS SAAVEDRA ${ }^{1, \mathrm{~b}}$, JORGE JIMÉNEZ ${ }^{2, \mathrm{c}}$
}

\section{Health and economic benefits of reducing $10 \mu \mathrm{m}$ particulate matter $\left(\mathrm{PM}_{10}\right)$ in Metropolitan Area of Concepción, Chile}

Background: Several international studies show the effects of $P M_{10}$ pollution on health but specific analyses for many cities in Chile are lacking. Aim: To relate $P M_{10}$ concentrations to effects with population health and quantify the economic benefits of its reduction in Concepción Metropolitan Area. Material and Methods: Poisson regression and generalized additive models were used to analyze the short-term effects of $P M_{10}$ on mortality and morbidity, controlling for lags, seasonal, trend and weather variables. The damage function method to determine the economic impact of pollution reduction was used. Results: The selected concentration-response $(C-R)$ coefficients showed that $P M_{10}$ concentrations had effects on hospital admissions with a two days lag for respiratory diseases in children under 15 years of age and with a one day lag for asthma in patients over 64 years. The effects on premature mortality had a six days lag. The decrease in $1 \mu \mathrm{g} / \mathrm{m}^{3}$ of $P M_{10}$ concentration would generate benefits ranging from 1,025.8 to 32,490.9 million of Chilean pesos per year, with a confidence level of $95 \%$, according the estimation based on concentration-response coefficients and their economic cost. Conclusions: Reduction of $P M_{10}$ would have important health and economic benefits.

(Rev Med Chile 2015; 143: 475-483) disease.

Key words: Air pollution; Mortality; Particulate matter; PM10; Respiratory

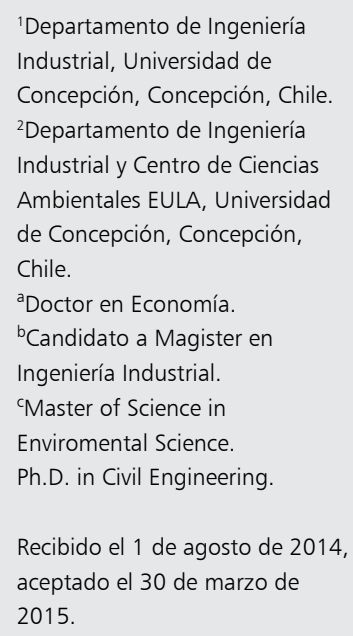

Recibido el 1 de agosto de 2014, aceptado el 30 de marzo de 2015.

Correspondencia a: Cristian Mardones P., Edmundo Larenas 219, Concepción, Chile. Teléfono: 56-41-2203614. crismardones@udec.cl

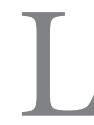

as mediciones en las estaciones de monitoreo llevaron a que en 2006 se declarara zona latente por material particulado respirable $\left(\mathrm{MP}_{10}\right)$ a Concepción Metropolitano; y que posteriormente, en el año 2011, se aprobara el Anteproyecto del Plan de Prevención Atmosférico para las comunas del Concepción Metropolitano, el cual incorpora medidas que buscan evitar que se supere la norma para este contaminante.

Esta situación es preocupante ya que diversos estudios internacionales han relacionado la mortalidad, los problemas cardiovasculares y las enfermedades respiratorias con la contaminación del aire ${ }^{1}$. También se ha investigado el material particulado fino y sus efectos sobre la morbilidad ${ }^{2}$ y mortalidad ${ }^{3}$. Sin embargo, no es sólo la concentración la que produce daños sino que también la composición afecta el estado de salud de las personas ${ }^{4}$. En Santiago de Chile, se logró asociar la muerte prematura a la contaminación vehicular ${ }^{5}$, y también se concluyó que las concentraciones "aceptables" para la mayoría de la población no resultan ser adecuadas para los más ancianos ${ }^{6}$. En la ciudad de Temuco también se encontró una 
asociación entre contaminación diaria por $\mathrm{MP}_{10}$ y mortalidad por causas respiratorias, cardiovasculares y cardiorrespiratorias ${ }^{7}$.

En este trabajo, los efectos de la concentración de $\mathrm{MP}_{10}$ sobre la mortalidad y morbilidad son estudiados de forma desagregada según causa y por rango etario con distintos modelos estadísticos. Luego, se valorizan económicamente los efectos en la salud de variaciones en las concentraciones diarias de $\mathrm{MP}_{10}$. Estos beneficios económicos provienen casi en su totalidad de las muertes prematuras evitadas, y en mucho menor magnitud, por el ahorro asociado al tratamiento de enfermedades respiratorias.

Específicamente, para la evaluación económica de los beneficios en salud se utiliza el método de la función de daño, el cual ha sido utilizado en Chile para evaluar diversos planes de descontaminación ${ }^{8}$ (Cifuentes L. Generación de Instrumentos de Gestión Ambiental para la Actualización del Plan de Descontaminación Atmosférica para la Región Metropolitana de Santiago al Año 2000. Santiago, Chile, Pontificia Universidad Católica de Chile. Departamento de Ingeniería Industrial y de Sistemas, Diciembre 2000 y DICTUC. Análisis General del Impacto Económico y Social de la norma de calidad del aire por $\mathrm{MP}_{2,5}$. Santiago 2010). Las etapas del método de la función de daño son las siguientes: las emisiones de las distintas fuentes contaminantes se traducen en concentraciones sobre una localidad; existe una población expuesta que sufre los efectos de las concentraciones ambientales; luego, para relacionar las concentraciones con los efectos en la salud, se utilizan las funciones concentración-respuesta (C-R); finalmente, se pueden calcular los beneficios generados a partir de los daños evitados en mortalidad prematura y morbilidad debido a la reducción en las concentraciones del contaminante.

\section{Material y Método}

\section{Estudio epidemiológico}

La relación entre los niveles de concentraciones y los efectos sobre la salud se establece a través de las funciones concentración-respuesta (C-R). Estas funciones se obtienen, por lo general, de estudios epidemiológicos de series de tiempo, de cohorte, o de sección transversal. Los estudios de series de tiempo relacionan estadísticamente los cambios temporales (por lo general diarios) en la incidencia de efectos en la población de una ciudad con los cambios en la concentración de un contaminante. La población expuesta a través del tiempo actúa como su propio control estadístico. Sin embargo, para establecer la función C-R se requiere condicionar por variables confundentes, como los datos meteorológicos, pues varían de forma diaria al igual que la contaminación. Dada la información disponible esta investigación tiene carácter de serie de tiempo.

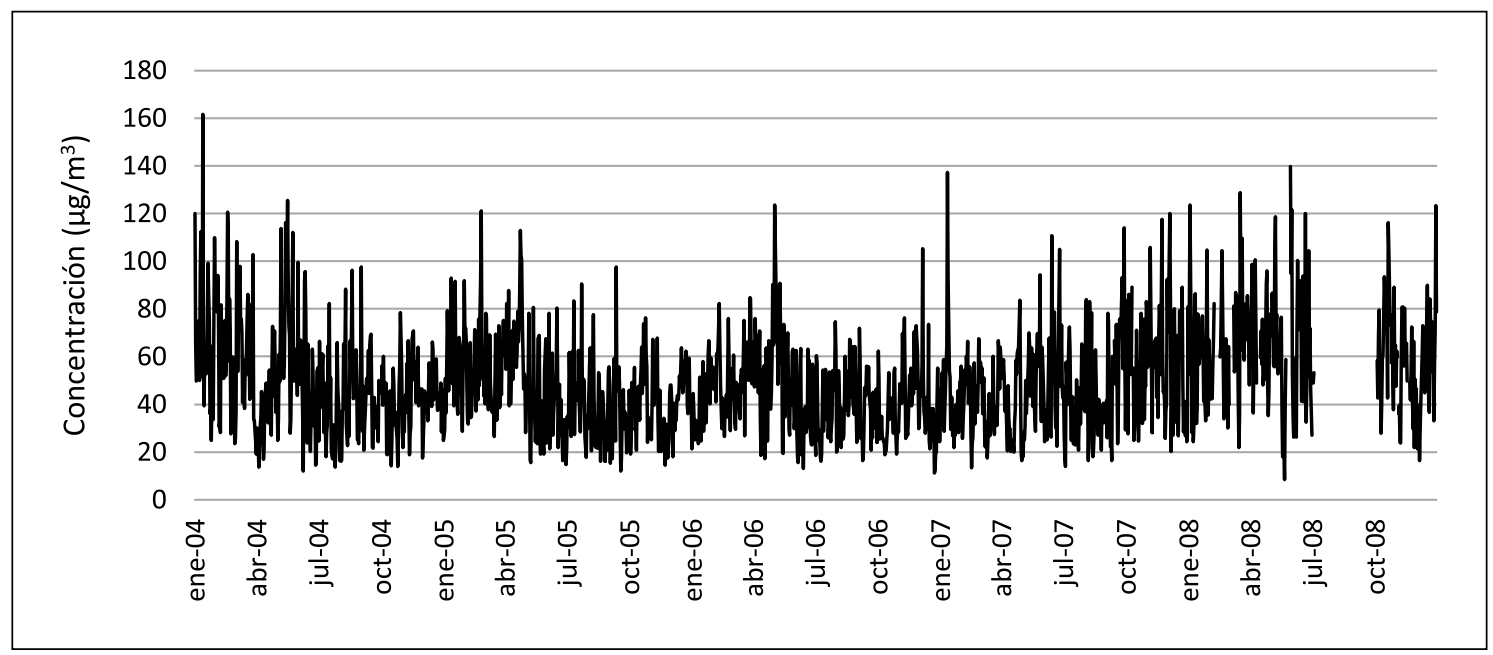

Figura 1. Concentraciones diarias $\mathrm{MP}_{10}$ Concepción Metropolitano 2004-2008. Fuente: Elaboración propia en base a SINCA. 
Para definir los grupos vulnerables, las causas de enfermedades y mortalidad prematura, confundentes, modelos estadísticos y consideraciones más frecuentemente aplicados, se realizó una revisión de distintas investigaciones epidemiológicas internacionales y nacionales, un resumen de las más relevantes se puede observar en la Tabla 1.

A partir de esta revisión se decidió utilizar los modelos de regresión Poisson y GAM (Modelo Aditivo Generalizado), también, se determinó que no existe un agrupamiento etario único en las distintas investigaciones, por lo cual se consideraron los grupos más frecuentemente utilizados, estos son mayores 64 años, de 64 a 15 años, y los menores de 15 años. Los efectos sobre la salud más estudiados son las muertes no accidentales (excluye sólo V00-Y98 del ICD-10), respiratorias (I00 a I99) y cardiovasculares (J00 a J99); que son incluidas en este estudio junto con las causas digestivas como control (K00 a K93). Además, se decidió analizar un nivel de desagregación mayor de enfermedades, que consideran asma (J45), bronquitis aguda (J20), enfermedades cardíacas isquémicas (I20 a I25) y cirrosis hepática alcohólica (K703), esta última también como control.

La información disponible junto con la revisión bibliográfica permitió definir las variables para realizar los análisis estadísticos (Tabla 2). Específicamente, se reunieron datos meteorológicos, concentraciones de $\mathrm{MP}_{10}$, ingresos hospitalarios y de muertes diarias ocurridas en Concepción Metropolitano.

Los datos meteorológicos fueron obtenidos de la Dirección Meteorológica de Chile (registrados en el aeropuerto Carriel Sur) e incluyen registros diarios de temperatura, humedad, velocidad del viento y lluvia.

La información de las concentraciones diarias de $\mathrm{MP}_{10}$ provienen de las estaciones de monitoreo del Sistema de Información Nacional de Calidad del Aire (SINCA) entre los años 2004 y 2008. Los datos diarios de mortalidad e ingresos hospitalarios por enfermedades, se obtuvieron del Departamento de Estadísticas e Información de Salud (DEIS) del Ministerio de Salud. En la Tabla 3 se presentan a modo de resumen los totales anuales para cada uno de los efectos estudiados.

Se puede observar que la mayor cantidad anual de casos de mortalidad se presenta en las personas mayores de 64 años, y que a mayor desagregación por tipo de causa existen menos casos. Para los ingresos hospitalarios por enfermedades respiratorias, la mayoría de casos son atribuidos a las personas menores de 15 años, mientras para los ingresos por enfermedades al sistema respiratorio y circulatorio, las personas mayores de 64 años presentan cifras similares al grupo entre 15 y 64 años, aunque representan una menor población.

Para generar una asociación entre el $\mathrm{MP}_{10}$ y los efectos en la salud se requieren utilizar modelos estadísticos. Los modelos de regresión Poisson pertenecen a los Modelos Lineales Generalizados (GLM) $\mathrm{y}$ se pueden representar de la siguiente forma:

$\log ($ efecto $)=\alpha_{0}+\sum_{i=1}^{n} \alpha_{i} *$ variable $_{i}+\beta * M P_{10}+e$

Por otra parte, los modelos GAM permiten evaluar efectos no lineales al incluir las variables explicativas como funciones alisadas llamadas splines. En este estudio son incluidas las variables meteorológicas en splines con tres grados de libertad, excluyendo la lluvia que tiene carácter binario. Estos modelos se puede representar como:

$\log ($ efecto $)=\alpha_{0}+\sum_{i=1}^{n-m} \alpha_{i} *$ variable $_{i}+\sum_{i=n-m}^{n} \alpha_{i} * f_{i}\left(\right.$ mete $\left._{i}\right)+\beta * M P_{10}+e$

Para la estimación de estos modelos se utiliza el protocolo APHEA (Air Pollution and Health European Approach) en el cual las variables de interés se incluyen de manera secuencial, en la medida que cumplan con tener un valor-p menor a un valor crítico (en este estudio es 0,15 ), luego si la variable asociada a la contaminación aún se conserva y tiene un valor-p menor a 0,05 se considera que existe una relación estadística entre la salud y la contaminación.

A partir del coeficiente C-R asociado al $\mathrm{MP}_{10}$ es posible estimar el riesgo relativo $\left(R R_{i j}\right)$ de la causa $i$ en el grupo de población $j$, el cual se calcula de la siguiente forma:

$$
R R_{i j}=\exp ^{\left(\beta_{i j}^{*} \Delta M P_{10}\right)}
$$

Para validar los coeficientes C-R calculados, se definieron algunos criterios de selección. Primero, sólo fueron seleccionados aquellos coeficientes que tuvieran un nivel de significancia menor a $5 \%$. Segundo, se escogieron aquellos coeficientes que arrojan un riesgo relativo (RR) entre 1 y 2 , ya que para fuera de este rango de valores no es razonable atribuir efectos de la contaminación sobre la salud. Finalmente, se puede incluir un criterio 


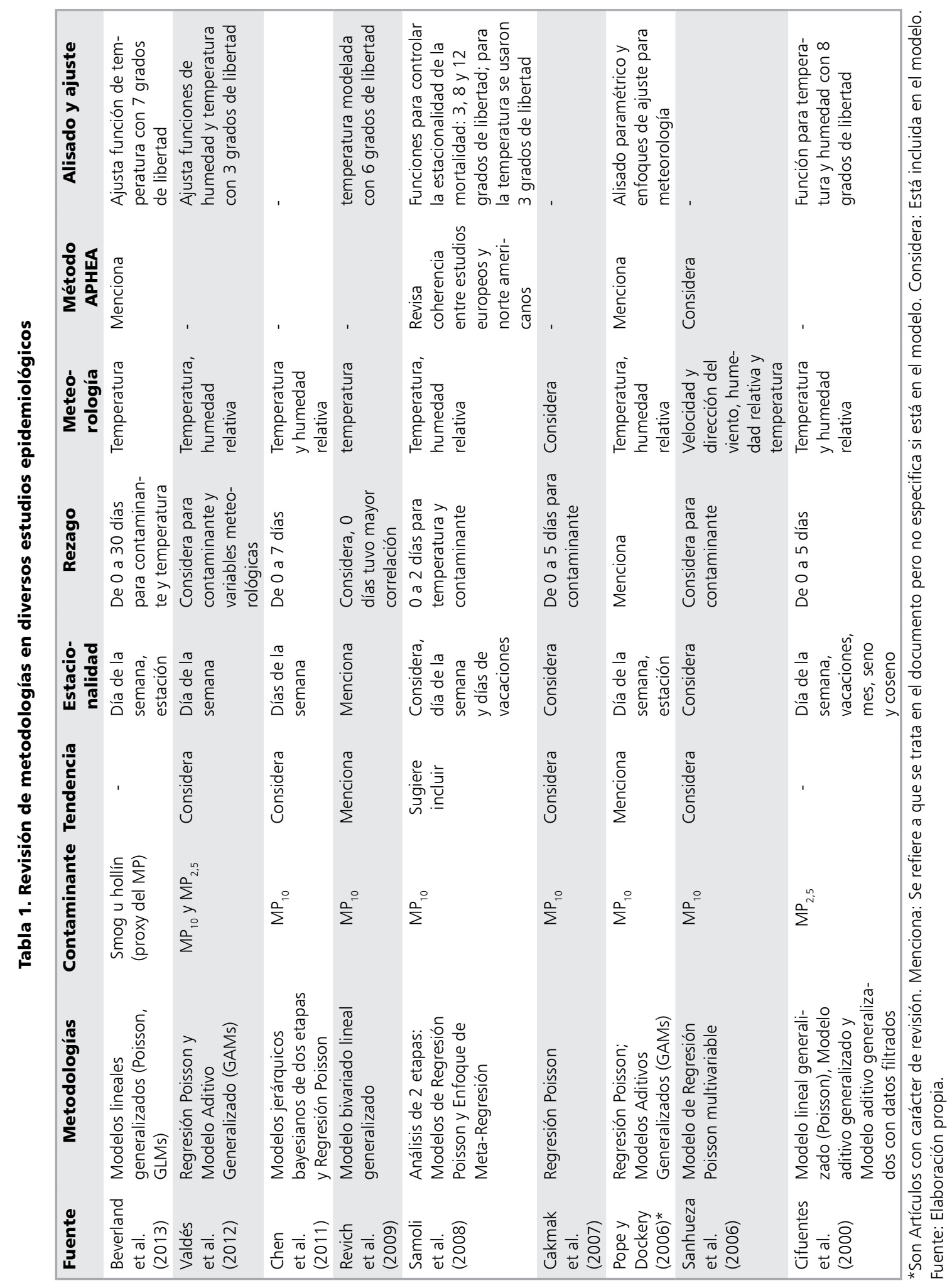


Tabla 2. Variables utilizadas en los modelos

\begin{tabular}{|c|c|c|}
\hline Carácter & Variable & Descripción \\
\hline Contaminante & MP10 & Concentraciones diarias de $\mathrm{MP}_{10}$ \\
\hline Rezagos & $\begin{array}{l}\text { MP10_1a1, MP10_1a2, MP10_1a3, } \\
\text { MP10_1a4, MP10_1a5, MP10_1a6, } \\
\text { MP10_1a7, MP10_1a8, MP10_1a9, } \\
\text { MP10_1a10 }\end{array}$ & Concentraciones de $\mathrm{MP}_{10}$ considerando rezagos de 1 a 10 días \\
\hline Meteorología & $\begin{array}{l}\text { temp* } \\
\text { humedad* } \\
\text { velviento* } \\
\text { lluvia }\end{array}$ & $\begin{array}{l}\text { Temperatura media diaria (grados Celsius) } \\
\text { Humedad relativa diaria }(\%) \\
\text { Velocidad promedio del viento diaria }(\mathrm{km} / \mathrm{h}) \\
0 \circ 1 \text {, si ha llovido o no ese día }\end{array}$ \\
\hline Estacionalidad & $\begin{array}{l}\text { feriado } \\
\text { I, } m, x, j, v, s, d \\
\text { ene, feb, mar, abr, may, jun, jul, } \\
\text { ago, sep, oct, nov, dic }\end{array}$ & $\begin{array}{l}\text { Si determinado día es feriado } \\
\text { Son los días de la semana } \\
\text { Meses del año }\end{array}$ \\
\hline Causas** & $\begin{array}{l}\text { na_q } \\
\text { i_q } \\
\text { j_q } \\
\text { k_q } \\
\text { isq_q } \\
\text { bro_q } \\
\text { asm_q } \\
\text { k703_q }\end{array}$ & $\begin{array}{l}\text { No accidental } \\
\text { Sistema circulatorio } \\
\text { Sistema respiratorio } \\
\text { Sistema digestivo } \\
\text { Isquemia del corazón } \\
\text { Bronquitis aguda } \\
\text { Asma } \\
\text { Cirrosis hepática alcohólica }\end{array}$ \\
\hline Tendencia & $\begin{array}{l}\mathrm{t} \\
\mathrm{t} \text {-2 } \\
\text { senk } \\
\text { cosk }\end{array}$ & $\begin{array}{l}\text { Controla la estacionalidad lineal de los datos } \\
\text { Controla la estacionalidad exponencial }\left(\mathrm{t}^{2}\right) \\
\text { Controla la estacionalidad sinusoidal de la forma } \sin (2 \mathrm{k} \pi \mathrm{t} / 365) \text {, } \\
\text { donde } \mathrm{k} \text { asume valores de } 1 \text { a } 6 \\
\text { Controla la estacionalidad cosenoideal de la forma } \cos (2 \mathrm{k} \pi \mathrm{t} / 365) \\
\text { donde } \mathrm{k} \text { asume valores de } 1 \text { a } 6\end{array}$ \\
\hline
\end{tabular}

*Para estas variables en los modelos GAM se van a introducir como si fueran funciones con 3 grados de libertad (g.l.). **Para las causas " $q$ " corresponderá a: tot (todos los casos), 15 (casos de menores de 15 años), 40 (casos entre 15 y 64 años) y 64 (casos de mayores de 64 años). Fuente: Elaboración propia.

adicional de exclusión en el caso de los ingresos hospitalarios, ya que si existen efectos para el cual se tiene más de un coeficiente C-R que cumple con el nivel de significancia y el rango de RR, pero que pertenecen a distintos niveles de desagregación, para no doble contabilizar los efectos se escoge aquél de mayor desagregación.

\section{Evaluación Económica de Beneficios en Salud}

Para el cálculo de beneficios se utiliza el método de la función de daño que consiste en una secuencia de modelos epidemiológicos y de valoración económica.

En primer lugar se utilizan modelos que vinculan los efectos de un cambio diario en la concentración de $\mathrm{MP}_{10}$ sobre la salud de la población expuesta. Así, las funciones C-R para mortalidad prematura y morbilidad estimadas se proyectan a toda la población, para ello se multiplican por sus respectivas tasas bases diarias y población expuesta por el período de un año. Las tasas bases diarias reflejan el número de casos ocurridos diariamente en Concepción Metropolitano. Así, al multiplicar cada tasa base diaria por su respectivo coeficiente $\mathrm{C}-\mathrm{R}$ y agregando los casos en el período de un año para todas las comunas es posible estimar los efectos sobre la salud ante la variación de $1 \mu \mathrm{g} / \mathrm{m}^{3} \mathrm{de} \mathrm{MP}_{10}$.

Luego, se requiere cuantificar en términos monetarios los daños en salud, es decir, los costos monetarios asociados a los efectos valorados, que en este caso incluyen el valor de la vida estadística (VVE) por los casos de mortalidad prematura y los costos monetarios por los ingresos hospitalarios.

Para asociar valores monetarios a la morta- 
Tabla 3. Casos anuales de mortalidad y morbilidad en Concepción Metropolitano desagregados por causa y edad 2004-2008

\begin{tabular}{|c|c|c|c|c|c|c|c|c|c|c|}
\hline $\begin{array}{l}\text { causas/ } \\
\text { año }\end{array}$ & 2004 & 2005 & $\begin{array}{l}\text { ortalidad } \\
2006\end{array}$ & 2007 & 2008 & 2004 & 2005 & $\begin{array}{l}\text { Morbilidad } \\
2006\end{array}$ & 2007 & 2008 \\
\hline na_tot & 4.104 & 4.953 & 4.823 & 5.444 & 5.154 & 99.558 & 93.637 & 98.234 & 98.393 & 92.214 \\
\hline na_15 & 143 & 140 & 140 & 157 & 134 & 19.686 & 18.441 & 18.964 & 17.620 & 16.704 \\
\hline na_40 & 1.330 & 1.541 & 1.531 & 1.652 & 1.643 & 61.532 & 57.647 & 60.262 & 60.659 & 56.785 \\
\hline na_64 & 2.631 & 3.272 & 3.152 & 3.635 & 3.377 & 18.340 & 17.549 & 19.008 & 20.114 & 18.725 \\
\hline i_tot & 1.147 & 1.386 & 1.374 & 1.531 & 1.420 & 7.153 & 7.167 & 7.199 & 7.571 & 7.030 \\
\hline i_15 & 0 & 5 & 0 & 3 & 2 & 159 & 207 & 131 & 121 & 129 \\
\hline i_40 & 240 & 277 & 277 & 293 & 286 & 3.566 & 3.699 & 3.585 & 3.667 & 3.509 \\
\hline i_64 & 907 & 1.104 & 1.097 & 1.235 & 1.132 & 3.428 & 3.261 & 3.483 & 3.783 & 3.392 \\
\hline j_tot & 356 & 379 & 393 & 550 & 428 & 12.298 & 11.793 & 11.489 & 12.409 & 9.712 \\
\hline j_14 & 4 & 3 & 2 & 5 & 2 & 6.235 & 5.526 & 5.535 & 5.547 & 4.474 \\
\hline j_40 & 47 & 41 & 47 & 83 & 55 & 3.079 & 3.323 & 2.984 & 3.264 & 2.558 \\
\hline j_64 & 305 & 335 & 344 & 462 & 371 & 2.984 & 2.944 & 2.970 & 3.598 & 2.680 \\
\hline k_tot & 430 & 461 & 448 & 507 & 499 & 12.545 & 12.221 & 12.481 & 12.317 & 12.341 \\
\hline k_15 & 3 & 2 & 2 & 1 & 0 & 2.184 & 2.156 & 1.972 & 1.711 & 1.719 \\
\hline$k \_40$ & 220 & 231 & 217 & 231 & 255 & 7.885 & 7.650 & 7.918 & 8.104 & 8.158 \\
\hline k_64 & 207 & 228 & 229 & 275 & 244 & 2.476 & 2.415 & 2.591 & 2.502 & 2.464 \\
\hline isq_tot & 360 & 444 & 384 & 453 & 393 & 1.502 & 1.615 & 1.310 & 1.294 & 1.104 \\
\hline isq_15 & 0 & 0 & 0 & 0 & 0 & 1 & 0 & 0 & 0 & 0 \\
\hline isq_40 & 94 & 109 & 94 & 115 & 111 & 759 & 852 & 676 & 679 & 599 \\
\hline isq_64 & 266 & 335 & 290 & 338 & 282 & 742 & 763 & 634 & 615 & 505 \\
\hline bro_tot & 7 & 4 & 2 & 5 & 3 & 1.281 & 856 & 804 & 1.254 & 671 \\
\hline bro_15 & 0 & 0 & 0 & 0 & 1 & 1.095 & 851 & 799 & 1.153 & 649 \\
\hline bro_40 & 0 & 0 & 0 & 0 & 1 & 78 & 5 & 1 & 44 & 10 \\
\hline bro_64 & 7 & 4 & 2 & 5 & 1 & 108 & 0 & 4 & 57 & 12 \\
\hline asm_tot & 12 & 9 & 14 & 14 & 14 & 232 & 223 & 235 & 351 & 237 \\
\hline asm_15 & 0 & 0 & 0 & 0 & 0 & 121 & 109 & 147 & 229 & 142 \\
\hline asm_40 & 2 & 2 & 2 & 5 & 3 & 77 & 84 & 73 & 99 & 66 \\
\hline asm_64 & 10 & 7 & 12 & 9 & 11 & 34 & 30 & 15 & 23 & 29 \\
\hline k703_tot & 101 & 104 & 80 & 74 & 87 & 64 & 28 & 32 & 14 & 19 \\
\hline k703_15 & 0 & 0 & 0 & 0 & 0 & 0 & 0 & 0 & 0 & 0 \\
\hline k703_40 & 68 & 73 & 57 & 53 & 63 & 42 & 20 & 23 & 11 & 15 \\
\hline k703_64 & 33 & 31 & 23 & 21 & 24 & 22 & 8 & 9 & 3 & 4 \\
\hline
\end{tabular}

Fuente: Elaboración propia en base a DEIS.

lidad prematura en la literatura se han utilizado típicamente tres enfoques: valoración contingente, salarios hedónicos y capital humano. Mientras que para estimar los costos asociados a la morbilidad, generalmente se han utilizado los costos directos asociados con la enfermedad, gastos defensivos y valoración contingente ${ }^{11}$.

En este trabajo para la valorización de la mortalidad prematura evitada se utilizan los resultados de un estudio de salarios hedónicos que a partir de 
diferencias salariales generadas por mayor riesgo de mortalidad laboral estima para Chile un VVE de 4,6 millones de dólares ${ }^{10}$. En cuanto a los casos evitados de morbilidad, estos son valorizados de acuerdo al costo de hospitalización que incluye el valor de los días cama más la consulta médica para cada enfermedad (MM\$ 0,319, MM\$ 0,226y MM\$ 0,168 para admisiones hospitalarias cardiovasculares, respiratorias y asma, respectivamente). Estos costos se calculan según el número de días de hospitalización y el valor de la prestación médica que proviene del "Estudio de costos de prestaciones de salud" realizado por el Departamento de Salud Pública de la Pontifica Universidad Católica de Chile (Pontificia Universidad Católica de Chile. Estudio de costos de prestaciones de salud. Pontificia Universidad Católica de Chile, Departamento de Salud Pública. Santiago 2012).

Finalmente, los beneficios monetarios en salud se calculan con la siguiente fórmula:

$\Delta$ Beneficios $=\sum_{j=1}^{m} \sum_{i=1}^{n}$ Valor unitario $_{i j} *$ Tasa base $_{i j} *$ Población $_{j} * \beta_{i j} * \Delta M P_{10}$

Donde,

Población: subgrupo $j$ de la población.

Tasa base ${ }_{i j}$ tasa base del período para el efecto tratado $i$ sobre el subgrupo de la población $j$.

$\beta_{i j}$ : coeficiente C-R calculado para el contaminante con respecto al efecto tratado $i$ sobre el subgrupo de la población $j$.

Valor ij: valorización del efecto tratado $i$ sobre el subgrupo de la población $j$.

\section{Resultados}

En esta sección se presentan los coeficientes $\mathrm{C}-\mathrm{R}$ estimados y que sirven para asociar los niveles de concentración de $\mathrm{MP}_{10}$ con las muertes e ingresos hospitalarios ocurridos en Concepción Metropolitano.

De acuerdo a los resultados de la Tabla 4 y los criterios de selección de la sección 2.1, se decidió utilizar para la valoración de beneficios en salud el coeficiente C-R calculado con la regresión Poisson para el total de muertes no accidentales, donde el efecto es producido por el $\mathrm{MP}_{10}$ con un rezago de seis días. Así como también, los coeficientes de los ingresos hospitalarios asociado a enfermedades respiratorias en menores de 15 años $\left(j_{-} 15\right)$ y asma en mayores de 64 años (asm_64). La especificación de los modelos seleccionados se presenta en la Tabla 5.

En la Tabla 6 se presentan los beneficios marginales en salud generados por la reducción de la concentración en una unidad. Para comprender el cálculo, se ejemplifica la estimación de beneficios para la comuna de Concepción: el coeficiente $\mathrm{C}-\mathrm{R}$ para mortalidad prematura $(0,001284)$ se multiplica por los habitantes de la comuna (230.729 personas), por la tasa de efectos base anual $(0,503 \%)$, por el VVE en millones de pesos (MM\$2.544,3) y por el cambio en las concentraciones $\left(1 \mu \mathrm{g} / \mathrm{m}^{3}\right)$. El valor obtenido son los beneficios monetarios por menor mortalidad en la comuna de Concepción (MM\$3.794,1), los cuales sumados a los beneficios por menor morbilidad

Tabla 4. Coeficientes C-R en Concepción Metropolitano

\begin{tabular}{|lllcllll|}
\hline Grupo & Caso & Modelo & Contaminante & Coef. C-R & D. Est. & Valor-p & RR \\
na_tot & Ingreso Hosp. & GAM & MP10_1A1 & 0,000233 & 0,00008 & 0,0035 & 1,0236 \\
na_15 & Ingreso Hosp. & Poisson & MP10_1A7 & 0,000930 & 0,00034 & 0,0069 & 1,0974 \\
na_15 & Ingreso Hosp. & GAM & MP10_1A1 & 0,000475 & 0,00018 & 0,0079 & 1,0486 \\
na_40 & Ingreso Hosp. & GAM & MP10_1A1 & 0,000214 & 0,00010 & 0,0344 & 1,0216 \\
j_tot & Ingreso Hosp. & GAM & MP10_1A2 & 0,000561 & 0,00027 & 0,0367 & 1,0577 \\
j_15 & Ingreso Hosp. & GAM & MP10_1A2 & 0,000900 & 0,00038 & 0,0183 & 1,0941 \\
asm_64 & Ingreso Hosp. & GAM & MP10_1A1 & 0,000938 & 0,00034 & 0,0060 & 1,0984 \\
na_tot & Mortalidad & Poisson & MP10_1A6 & 0,001284 & 0,00062 & 0,0366 & 1,1370 \\
\hline na_tot & Mortalidad & GAM & MP10_1A6 & 0,001194 & 0,00060 & 0,0195 & 1,1269 \\
\hline isq_64 & Mortalidad & Poisson & MP10_1A4 & 0,000645 & 0,00024 & 0,0065 & 1,0666 \\
\hline
\end{tabular}

Fuente: Elaboración propia. 
Tabla 5. Especificación de los modelos de regresión seleccionados para la valoración de beneficios en salud en Concepción Metropolitano

\begin{tabular}{|c|c|c|c|c|c|}
\hline Gupo & Caso & Método & Tendencia & Estacionalidad & Meteorología \\
\hline j_15 & Ingreso Hosp. & GAM & t_2 & $\begin{array}{l}\operatorname{sen} 1 \operatorname{sen} 2 \operatorname{sen} 3 \operatorname{sen} 4 \operatorname{sen} 6 \cos 1 \cos 4 \cos 6 \\
\text { feriado I } \mathrm{m} \text { x j v s mar abr may jun sep nov } \\
\text { verano }\end{array}$ & $\begin{array}{l}\text { temp (3 g.l.) } \\
\text { humedad (3 g.l.) } \\
\text { velviento ( } 3 \text { g.l.) }\end{array}$ \\
\hline asm_64 & Ingreso Hosp. & GAM & $t$ & $\operatorname{sen} 3 \mathrm{~s}$ & temp (3 g.I.) \\
\hline na_tot & Mortalidad & Poisson & $\mathrm{tt} \_2$ & $\operatorname{sen} 1 \cos 1 \cos 2 \cos 4 \times$ feb & Iluvia \\
\hline
\end{tabular}

Fuente: Elaboración propia.

estimados con similar procedimiento (MM\$ 0,003 por asma en mayores de 65 años y MM\$ 0,205 por enfermedades respiratorias en menores de 15 años) permiten estimar beneficios totales en la misma comuna por MM\$3.794,3.

Es importante mencionar, que como los coeficientes $\mathrm{C}-\mathrm{R}$ provienen de cálculos estadísticos, estos tienen un intervalo de confianza, por lo que también es posible obtener un intervalo para los beneficios (límite inferior y superior). Como era de esperar las comunas que presentan mayores beneficios son Concepción y Talcahuano, esto se debe a que los beneficios en salud están directamente relacionados con la población afectada, cuyo número es mayor en estas comunas.

\section{Discusión}

En general el cálculo de los beneficios en salud es sensible a los coeficientes C-R utilizados (especialmente de mortalidad), ya que para los otros parámetros requeridos en la función de daño existe menos incertidumbre respecto a su valor (población afectada, tasas base y variación en la contaminación).

En particular, para este estudio el coeficiente C-R de mortalidad para el total de la población por exposición aguda de $\mathrm{MP}_{10}$ tiene un valor de 0,0012 y 0,0013 , dependiendo del modelo estadístico utilizado (GAM o Poisson), valor similar al encontrado en un estudio para la ciudad de Santiago que arrojó un 0,0012 para exposición aguda a $\mathrm{MP}_{2,5}{ }^{5}$, cabe señalar que en la zona de estudio un alto porcentaje del $\mathrm{MP}_{10}$ corresponde a $\mathrm{MP}_{2,5}$. A diferencia de otro estudio para Santiago ${ }^{11}$ que encuentra efectos significativos del $\mathrm{MP}_{2,5}$ sobre todas las causas con nivel de desagregación media para
Tabla 6. Beneficios marginales estimados (MM\$)

\begin{tabular}{|lrrr|}
\hline Comuna & $\begin{array}{c}\text { Límite } \\
\text { inferior }\end{array}$ & Medio & $\begin{array}{c}\text { Límite } \\
\text { superior }\end{array}$ \\
\hline Concepción & 232,3 & 3794,3 & $7.356,6$ \\
\hline Coronel & 113,5 & 1853,7 & $3.594,1$ \\
\hline Chiguayante & 138,0 & 2254,1 & $4.370,4$ \\
\hline Hualqui & 23,7 & 387,0 & 750,3 \\
\hline Lota & 47,0 & 768,1 & $1.489,3$ \\
\hline Penco & 55,6 & 908,5 & $1.761,4$ \\
\hline San Pedro de la Paz & 102,4 & 1672,7 & $3.243,1$ \\
\hline Talcahuano & 172,3 & 2814,5 & $5.457,0$ \\
\hline Tomé & 57,2 & 934,7 & $1.812,2$ \\
\hline Hualpén & 83,9 & 1370,2 & $2.656,6$ \\
\hline Total & $1.025,8$ & $16.757,7$ & $32.490,9$ \\
\hline
\end{tabular}

Fuente: Elaboración propia.

la mortalidad, en el presente estudio se encuentra un efecto sobre el total de causas no accidentales, y en causas isquémicas al corazón en mayores de 64 años, esto último concuerda parcialmente con quienes encuentran un efecto significativo de la mortalidad sobre la población de mayor edad ${ }^{6}$.

De acuerdo a los datos y metodología utilizada, los beneficios en salud estimados para todas las comunas del Concepción Metropolitano varían entre 1.025,8 y 32.490,9 millones de pesos anuales con un nivel de confianza de $95 \%$ por una reducción de $1 \mathrm{mg} / \mathrm{m}^{3}$ en las concentraciones diarias de $\mathrm{MP}_{10}$, los cuales por los datos y metodología empleada corresponden a beneficios atribuibles a exposiciones de corto plazo.

Si se comparan los beneficios calculados en este estudio respecto a los que se podrían estimar 
con funciones $\mathrm{C}-\mathrm{R}$ reportados por la evidencia internacional de los efectos de largo plazo de las concentraciones de $\mathrm{MP}_{10}$ sobre la mortalidad en toda la población ${ }^{12}$, ajustando por la relación $\mathrm{MP}_{10}$ a $\mathrm{MP}_{2,5}$ en la zona de estudio, sería posible determinar beneficios anuales entre 19.520,5 y 142.281,1 millones de pesos en la zona del Concepción Metropolitano. La diferencia se debe principalmente a que los beneficios están influenciados casi en su totalidad por el efecto de la contaminación sobre la mortalidad y el coeficiente C-R para exposición a largo plazo es aproximadamente cinco veces mayor.

\section{Referencias}

1. Dockery DW, Pope CA, Xu X, Spengler JD, Ware JH, Fay ME, et al. An Association between Air Pollution and Mortality in Six U.S. Cities. N Engl J Med 1993; 329: 1753-9.

2. Pope CA, Dockery DW. Health Effects of Fine Particulate Air Pollution: Lines that Connect. J Air Waste Manag Assoc 2006; 56: 709-42.

3. Pope CA, Ezzati M, Dockery DW. Fine-Particulate Air Pollution and Life Expectancy in the United States. N Engl J Med 2009; 360: 376-86.

4. Polichetti G, Cocco S, Spinali A, Trimarco V, Nunziata A. Effects of particulate matter (PM10, PM2.5 and PM1) on the cardiovascular system. Toxicology 2009; 261: 1-8.

5. Cifuentes LA, Vega J, Köpfer K, Lave LB. Effect of the Fine Fraction of Particulate Matter versus the Coarse Mass and Other Pollutants on Daily Mortality in Santiago, Chile. J Air Waste Manag Assoc 2000; 50 (8): 1287-98.

6. Cakmak S, Dales RE, Vidal CB. Air Pollution and Mortality in Chile: Susceptibility among the Elderly. Environ Health Perspect 2007; 115 (4): 524-7.

7. Sanhueza P, Vargas C, Mellado P. Impacto de la conta- minación del aire por $\mathrm{PM}_{10}$ sobre la mortalidad diaria en Temuco. Rev Med Chile 2006; 134: 754-61.

8. Sánchez J, Valdés S, Ostro B. Estimación de los beneficios en salud del Plan de descontaminación de Santiago. Trim Econ 1998; LXV (3): 363-406.

9. Freeman III M. The measurement of environmental and resource values, theory and methods. Washington, D.C.: Resources for the Future; 2003.

10. Parada-Contzen M, Riquelme-Won A, Vásquez-Lavin, F. The value of a statistical life in Chile. Empir Econ 2012; 1-15.

11. Valdés A, Zanobetti A, Halonen J, Cifuentes L, Morata D, Schwartz J. Elemental concentrations of ambient particles and cause specific mortality in Santiago, Chile: a time series study. Environ Health 2012; 11: 82.

12. Pope CA, Burnett RT, Thun MJ, Calle EE, Krewski D, Thurston GD. Lung cancer, cardiopulmonary mortality, and long-term exposure to fine particulate air pollution. JAMA 2002; 287: 1132-41.

13. Beverland I, Carder M, Cohen G, Heal M, Agius R. Associations between short/medium-term variations in black smoke air pollution and mortality in the Glasgow conurbation, UK. Environ Int 2013; 62: 126-32.

14. Chen R, Kan H, Chen B, Huang W, Bai Z, Song G. Association of Particulate Air Pollution With Daily Mortality-The China Air Pollution and Health Effects Study. Am J Epidemiol 2011; 175 (11): 1173-81.

15. Revich B, Shaposhnikov D. The effects of particulate and ozone pollution on mortality in Moscow, Russia. Air Qual Atmos Health 2009; 3: 117-23.

16. Samoli E, Peng R, Ramsay T, Pipikou M, Touloumi G, Dominici F. Acute Effects of Ambient Particulate Matter on Mortality in Europe and North America: Results from the APHENA Study. Environ Health Perspect 2008; 116: 1480-6.

17. Pope CA, Burnett RT, Thurston GD, Thun MJ, Calle EE, Krewski D, et al. Cardiovascular mortality and longterm exposure to particulate air pollution: Epidemiological evidence of general pathophysiological pathways of diasease. Circulation 2004; 109: 71-7. 